\title{
De-implementation of detrimental feeding practices: a pilot protocol
}

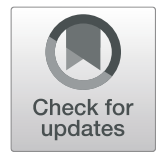

Taren Swindle 1* $^{*}$, Julie M. Rutledge ${ }^{2}$, Susan L. Johnson ${ }^{3}$, James P. Selig ${ }^{4}$ and Geoff M. Curran ${ }^{5}$

\begin{abstract}
Background: Early childhood educators (ECEs) often use detrimental feeding practices and are slow to implement positive feeding practices. Nevertheless, few studies have aimed to understand and change ECEs' feeding practices. This gap needs to be addressed because implementation (i.e., adding new, evidence-based practices) and deimplementation (i.e., stopping low-value or harmful practices) are distinct processes that require unique strategies.

Methods: We will develop a de-implementation strategy for detrimental feeding practices using evidence-based quality improvement (EBQI) sessions to engage stakeholders and draw on the Niven process model for deimplementation. Then, we will investigate the effects of the de-implementation strategy in a proof-of-principle study. The de-implementation strategy will be evaluated in 2 partnering childcare agencies using a pre-post, withinsite design. For our primary outcome, we will interview educators throughout the school year to assess the feasibility and acceptability of the intervention and survey them with standard measures for assessing feasibility and acceptability. For secondary outcomes, we will investigate its effects on the use of detrimental and evidence-based feeding practices by teachers and impacts on child BMI and diet.

Discussion: The current study will establish the feasibility and acceptability of our de-implementation approach and will provide preliminary data toward 3 predicted secondary outcomes: (1) decreased detrimental feeding practices by ECEs, (2) increased adoption of and fidelity to nutrition promotion practices, and (3) improved child dietary outcomes. These results are expected to contribute to the uptake and sustainability of mealtime interventions to improve the diets of young children. Results will also apply to the field of implementation science by informing processes for developing de-implementation approaches in a community setting.
\end{abstract}

Keywords: De-implementation, Childcare, Feeding practices, Nutrition, Implementation science

\section{Background}

Diet quality predicts a child's physical, mental, and academic well-being [1-3], yet the diets of many children in the USA are of low nutrient density $[4,5]$. One important setting for addressing children's dietary quality is childcare given that 11 million children under age 5 are in childcare settings in the USA [6] and may consume over two-thirds of their daily diets in this setting [7]. Research supports that childcare influences children's diets more broadly

\footnotetext{
* Correspondence: tswindle@uams.edu

'Department of Family and Preventive Medicine, University of Arkansas for Medical Sciences, 4301 W. Markham St, \#530, Little Rock, AR 72205-7199, USA Full list of author information is available at the end of the article
}

with spillover effects into the home [8, 9]. Further, dietary habits established in childhood persist into adulthood [10-12]. Thus, promoting healthy habits at childcare has both immediate and lifelong consequences.

Early childhood educators (ECEs) are influential adults in the childcare setting. Evidence-based feeding practices performed by ECEs that positively influence a child's diet include role modeling [13-16], cuing children to hunger and satiety [13, 14, 16-18], discussing and offering new foods [19-21], and facilitating the exploration of foods $[22,23]$. These practices are associated with children's increased self-regulation, fewer rejections of healthy foods, and increased willingness to try new foods [14, 
24-27]. These findings illustrate the positive impact adults can have on children when evidence-based feeding practices are implemented.

Detrimental feeding practices have also been identified $[14,15,24,28]$, and unfortunately, ECEs often use detrimental feeding practices and are slow to implement positive ones [19, 29, 30]. Detrimental feeding practices observed in childcare include hurrying children through the meal $[13,31]$ and coercing them to eat more [13, 31-36]. Observational research on childcare meals reveals that ECEs frequently pressure children to eat more food regardless of hunger state (19\%), hurry children to finish or "be done" (32\%), and coerce children to eat certain foods (30\%) [18]. Detrimental practices are associated with overeating, long-term food rejections, and decreased intake of healthy foods [24, 29, 37]. Additional effects of detrimental feeding practices include higher child weight-for-height percentiles [38], diminished selfregulation $[14,25,39]$, and decreased preference for and intake of fruits and vegetables [21, 24, 32, 39]. The frequency of such detrimental feeding practices and their role in driving unhealthy eating behaviors indicate a need for intervention.

Reports by ECEs reveal that there is wide variation in the frequency and content of training about nutrition and feeding practices, how the training is delivered, and the qualifications of instructors [40-42]. Previous interventions that targeted ECEs include tailored training and provision of resources (e.g., policies and curricula) [43], short didactic courses [44], and web-based selfassessment tools with tailored recommendations and supports [45]. No studies have documented a significant reduction in the observed use of detrimental feeding practices by ECEs. Further, although accredited bodies have established guidelines for structuring meals (e.g., family-style dining and child-sized utensils) [13] and informing the goals of meals (e.g., promoting children's choice) [46], there are no similar guidelines to address the prevalent, detrimental feeding practices of ECEs. Therefore, a critical barrier to success in this area is the lack of effective strategies to stop detrimental practices.

Implementation Science (IS) provides a promising approach to address the need to reduce, remove, and/replace, detrimental feeding practices in children. Inherent to IS is the concept of stopping practices that are not evidence-based, particularly those that are harmful or costly-this is the goal of a de-implementation [47]. Such an approach has been applied to medical service overuse with the aims of reducing low-value services and overtreatment and increasing the use of evidencebased guidelines [48, 49]. De-implementing detrimental practices is distinct from implementing evidence-based practices [50]; the process can be more difficult and require more intense strategies [50]. De-implementation "is far more than merely reversing the implementation process" [51]. While medical communities have developed low-value lists (e.g., Choosing Wisely [51-53]) to prioritize patient-care practices that are ineffective or harmful $[48,54]$, simply disseminating recommendations for de-implementation has limited impact [55]. An added intervention is usually needed [56]. In clinical practice, the most effective strategies for deimplementation are new policies and changes to funding structures [56]. With ECEs' feeding, however, these types of top-down, control-based extrinsic motivators are not a realistic approach because ECEs feeding practices are not highly monitored and because these settings are often under-resourced. Rather, effective strategies to shift intrinsic motivation are likely needed. Thus, research is necessary to formulate effective deimplementation strategies in a community setting.

Previous studies by the study team highlight both individual-level and contextual factors that impact ECE feeding practices. For example, detrimental practices are more common in a cafeteria setting with crowded, inflexible schedules and in the presence of competing foods from home [57]. Additionally, teachers who use detrimental feeding practices frequently are also significantly less likely to adhere to other nutrition promotion practices (i.e., hands-on exposures). Interviews conducted with ECEs explored how cultural factors (both personal and professional) impact feeding practices with children and identified over 20 discrete barriers and facilitators, including a prevalent concern about child food insecurity that educators felt responsible to address, an emphasis on manners, and varying beliefs about encouraging children to try foods [58]. These data provide a solid understanding of factors influencing feeding practices from which to work to remove detrimental practices.

\section{Study aims}

In an ongoing trial, we are applying principles of Implementation Science (IS) to increase ECE adoption of evidence-based nutrition promotion practices through the WISE (Together, We Inspire Smart Eating) intervention (described below in detail). However, the ongoing study does not explicitly attempt to decrease routinized, detrimental feeding practices. This gap needs to be addressed because implementation (adding new, evidencebased practices) and de-implementation (stopping lowvalue or harmful practices) are distinct processes that require unique strategies $[50,51]$. We propose that each may independently predict eating behaviors. The planned study will explore an innovative deimplementation approach aimed to reduce detrimental feeding practices by ECEs. This is distinct from ongoing work aimed at increasing implementation of WISE 
evidence-based practices without attention to de-implementation [59].

Specific aim 1. Develop a de-implementation approach for detrimental feeding practices. We will use evidencebased quality improvement (EBQI) sessions [60-62] to engage stakeholders (parents, educators, and administrators) to develop a socio-culturally informed deimplementation approach for detrimental feeding practices. Qualitative data on barriers to evidence-based feeding practices and facilitators of detrimental ones (collected prior) will inform stakeholder-selected strategies.

Specific aim 2. Investigate the feasibility, acceptability, and preliminary effects of the de-implementation approach in a proof-of-principle study. The deimplementation approach will be evaluated in 2 partnering childcare agencies using a pre-post, within-site design [63]. The primary outcome will be feasibility and acceptability, which we will assess through qualitative interviews and educator surveys. Second outcomes will include measures of preliminary effectiveness including effects on the use of detrimental and evidence-based feeding practices by teachers and impacts on child body mass index (BMI) and diet. We will combine these data with data from an ongoing trial [59] to compare (a) basic WISE implementation, (b) enhanced WISE implementation, and (c) enhanced WISE implementation with deimplementation support that targets detrimental feeding. This is the focal aim of our study.

\section{Methods}

\section{Aim}

This project will enhance the overall objective of an ongoing trial [59] to develop and test implementation strategies that are designed to increase the adoption of 4 evidence-based components of the WISE intervention, which aims to prevent obesity and promote nutrition among children. Our data suggest that positive feeding practices is the only component of WISE that competes against detrimental, routinized practices. Thus, this study will pursue a de-implementation approach to decrease detrimental feeding practices.

\section{Setting and participants}

Lincoln Parish Head Start (15 classrooms) is primarily African American (ECEs, 97\%; families served, 87\%). Lincoln Parish Early Childhood Center (LPECC, 12 classrooms) serves families who are white (52\%), African American (40\%), or Hispanic (5\%). ECEs at LPECC are white (58\%) and African American (42\%).

\section{Intervention}

The WISE components [64] include (1) multiple handson exposures to fruits and vegetables, (2) use of a mascot puppet to promote fruits and vegetables to children, (3) appropriate role modeling by ECEs, and (4) positive ECE feeding practices. WISE lessons occur during classroom instruction time, and the training encourages ECEs to use WISE components 2 through 4 at meals as appropriate. The focus of the current study is on developing and testing strategies to decrease detrimental feeding practices which are hypothesized to compete with positive feeding practices.

\section{Design}

Our proposed method to develop and test a deimplementation approach to reduce detrimental feeding practices in the childcare setting will draw on (1) the Niven model of de-implementation [56], that proposes an iterative method by which stakeholders and practitioners partner to tailor, evaluate, and sustain a deimplementation approach; (2) salient theoretical domains in behavior change theories for designing implementation strategies [65] that draw on theories of motivation (e.g., social learning theory), action (e.g., cognitive behavioral theory), and organization (e.g., diffusion theory) and that align well with the categories of possible implementation strategies outlined by Powell et al. [66]; and (3) the RE-AIM evaluative framework [67], that provides a framework to assess outcomes of implementation and to guide measurements [68]. The integration of these approaches will provide a sound foundation for the proposed research.

\section{Aim 1}

Engaging stakeholders in the selection and tailoring of deimplementation strategies to comprise a comprehensive approach is central to the Niven process model of deimplementation [56]. We are operationalizing this process with an EBQI panel approach [60-62], which will operate according to principles of community-based participatory research [69] and best practices for engaging stakeholders in IS [70]. Directors, ECEs, and parents will contribute local knowledge needed to tailor strategies to their own contexts, while implementation experts (i.e., the research team) will contribute knowledge on materials, procedures, and tools needed for successful implementation. All members share decision-making power about prioritizing barriers and selecting implementation strategies to target those barriers. EBQI panels have been used to produce effective implementation strategies [71-73], while also promoting buy-in from implementing organizations and fostering beneficial partnerships between implementers and researchers [74].

The EBQI process will (1) prioritize barriers and facilitators to stopping detrimental feeding, (2) identify theoretically informed de-implementation strategies and match to priorities, and (3) tailor de-implementation 
strategies to the early childhood context. The panel will consider strategies that will promote sustainability to prevent reversion to detrimental feeding practices [56]. The panel will include at least 8 stakeholders from the partnering agencies and 2 stakeholders from an existing EBQI panel who will act as mentors to other stakeholders as needed. The diversity of the EBQI will reflect the diversity of the local context.

EBQI is a flexible process conducted across a series of meetings with topic-driven agendas; each session will last 2 hours. In EBQI session 1, the research team will present a summary of findings related to school-based feeding practices from the ongoing trial, conduct a "member checking" exercise with participants to check the validity of these findings, and reach consensus on key barriers and facilitators that will drive selection of the de-implementation strategies. In EBQI session 2, we will present potential implementation strategies mapped by the research team to the Expert Recommendations for Implementing Change (ERIC) [75] and taxonomy of implementation strategies with consideration of the theoretical domains of behavior change [65]. To reach a consensus on the implementation strategies, we will use techniques outlined by Powell et al. [76], including concept mapping. This method provides quantifiable information and promotes efficient collection of input in real time. In EBQI session 3, we will present the draft strategies/tools, collect feedback for revisions, and receive final approval to pilot test them. The research team will take 1 month to develop and finalize the strategies/tools selected by the group. In EBQI sessions 4, we will launch a pre-test of the materials in a small number of classrooms in which the stakeholders teach. In EBQI session 5 , we will gather feedback from stakeholders about the feasibility and acceptability of the de-implementation strategy to inform iterations and improvements to the approach.

\section{Aim 2}

After developing the de-implementation approach with stakeholders (aim 1), the next step in the Niven model of de-implementation [56] is to evaluate the process and impact of the approach. During summer professional development days, a face-to-face five hour training will be held with the ECEs, administrators, and key staff of the centers. The training will be led by the lead study investigators. The training will focus on teaching the attendees about the new approach that is developed from the EBQI sessions and practicing with the materials.

Using the RE-AIM evaluation framework (see Table 1), we will collect data focused on de-implementation outcomes as our primary outcome. Specifically, we will collect surveys, which will include the Acceptability of Intervention Measure (AIM) [77] and Feasibility of Intervention Measure (FIM) [77]. Although standardized thresholds for these measures have not been established, we will set our threshold for defining success as a mean of 4 or higher on the 5-point scale. Next, we will collect interviews with a subset of educators about their perceptions of the feasibility/acceptability of the deimplementation strategies as well as the feasibility/acceptability of cessation of detrimental feeding practices. This subset will include a purposive sample of educators based on their survey responses; we will randomly select from the top and bottom 25\% teachers on the FIM scores to identify interview participants. We expect to interview 5 educators from each group. This will provide an in-depth and well-rounded perspective on feasibility in our study. Other measures collected in alignment with Re-AIM will serve as secondary outcomes. Effectiveness focuses on determining for whom the approach had positive impacts. We will assess child BMI and child dietary intake using Resonance Raman Spectroscopy (RRS) [78-83] to measure carotenoid intake and parent report of intake of WISE foods as well as nutrient-poor food consumption within the last month using a modified Food Frequency Questionnaire (FFQ) based on validated measures [84-86]. A passive consent process is approved for the ongoing trial to collect child BMI and RRS; we expect to follow the same protocol for this study and collect these data for up to 540 students. RRS will provide an objective measure of carotenoid intake [87], and analyses will use this as a within- and not between-person comparisons (i.e., avoiding comparisons across different skin pigmentations). This non-invasive method has been validated in preschoolers [79] and has several logistical advantages over invasive measures such as high-performance liquid chromatography in the

Table 1 Outcome measures to evaluate de-implementation approach

\begin{tabular}{lll}
\hline Construct & Instruments & Data collected \\
\hline Effectiveness & BMI, RRS, FFQ & Impact on child weight and diet outcomes \\
De-adoption & $\begin{array}{l}\text { Table Talk, BMER; AFC } \\
\text { Strategies and Beliefs }\end{array}$ & $\begin{array}{l}\text { Total number of detrimental and evidence-based feeding practices; scales include coercive control } \\
\text { strategies, bribery with sweet foods, autonomy undermining, and social comparisons }\end{array}$ \\
$\begin{array}{ll}\text { De- } \\
\text { implementation }\end{array}$ & Wise Fidelity; Qualitative & Formative qualitative interviews to asses feasibility and acceptability of the de-implementation strat- \\
Maintenance & AFC, Table Talk, BMER & Sustained impact on reported and observed behavior
\end{tabular}


community setting (e.g., higher participation, no trained phlebotomist required). Research suggests that, even among ethnically diverse samples, RRS can be an effective measurement tool when conducted on the palm of the hand [78]. For FFQ, all parents will be sent a form inviting them to participate. We will contact parents who respond for participation. Based on previous efforts with this approach, we expect to recruit 200 parents to complete FFQ assessments.

De-adoption will be assessed with Table Talk [88] and the Food Intake module of the Building Mealtime Environments and Relationships (BMER) [89] inventory. These tools specifically measure the detrimental feeding practices targeted for de-implementation, as well as evidence-based ones targeted for adoption. Both are suited for live observations to record actual ECE behavior. These observations will occur three times across the intervention school year (fall, winter, spring) and once during the maintenance school year (fall). Each class (lead and assistant ECE) is observed twice at each observation period-once during a WISE lesson and once during the lunch mealtime for a total of 8 observations per class. Observers arrive in time to reduce reactivity before the observation time and then stay throughout the duration of the observation period (i.e., until the WISE lesson ends or the last child has the opportunity to eat).

Additionally, we will collect the About Feeding Children (AFC) Mealtime Strategies and Beliefs survey [90], a self-report measure of ECEs' beliefs and intended practices. The survey includes scales to assess verbal ("I tell the children if they have not eaten enough") and structural (e.g., classroom rule that children must take at least one bite) strategies that ECEs use when feeding children. In analyses, we will examine differences for ECEs who participated in both the EBQI sessions and the full-scale intervention.

Training protocols for the observational assessments (Table Talk and BMER) were refined in our previous work [88, 91]. First, data collectors complete a 2-h, inperson training that describes the logistics of completing observations, including discrete integration into the classroom visits to minimize reactivity. Second, data collectors learn about the intent of each observational item and code a video of a lunch as a group. Third, data collectors watch and code videos (at least 4 additional) on their own and review their answers with the PI or project RA, who have detailed answer keys. Data collectors must demonstrate $85 \%$ reliability against a video observation and live observation with the PI or project RA (i.e., established gold standard observers).

Implementation focuses on quality of delivery of and understanding why certain results were achieved (i.e., feasibility). To assess implementation, we will use the
WISE fidelity measure [64]. This instrument is rated on a 1 to 4 scale, with 4 representing the highest level of fidelity. Each core component is assessed with 2 items, and overall scores on the fidelity form are created by summing scores across items. De-implementation strategy feasibility and acceptability will be assessed through surveys with the AIM and FIM with all participants as well as formative qualitative interviews with selected ECEs in Spring $2020(N=10)$ [92].

Maintenance is how well intervention components and their effects are maintained. Maintenance of the cessation of detrimental feeding and use of evidence-based feeding practices will be assessed in the subsequent school year. This is important as preventing reversal to routine habits is a challenge for de-implementation efforts [51]. We will be able to model maintenance variability.

\section{Analysis \\ Aim 1}

We will use an online platform and database server to collect and store the EBQI panel's ratings of importance and feasibility. In real time, we will query the database [93] to plot potential strategies by their rated importance $(x$-axis) and feasibility ( $y$-axis) [76]. Strategies above the mean for both criteria will be considered for inclusion in the de-implementation approach.

After each EBQI session, research team members will write memos documenting what they observed and heard, what was resolved, and what remains undecided. The research team will discuss these and meeting minutes to guide the subsequent EBQI sessions and to inform development of the de-implementation training and support materials. The research team will also assimilate panel feedback, translate it to actionable plans, and develop the next iteration of materials that need input from the panel. Qualitative information from meeting minutes and audio recordings will be analyzed using directed content analysis [94] relative to the main goals of the EBQI process (e.g., matching barriers/facilitators to de-implementation strategies). After data from the final EQBI meeting are analyzed, the de-implementation approach will be ready for the proof-of-principle study in Aim 2.

\section{$\operatorname{Aim} 2$}

To assess the feasibility and acceptability of our deimplementation approach, transcripts of qualitative interviews with ECEs will be coded [95]. Two coders will independently analyze the content and then resolve any differences together. These findings and feedback from the EBQI panel will be used to optimize the deimplementation approach in an iterative fashion to 
arrive at a fully-specified approach for a future largescale trial.

Descriptive statistics on acceptability and feasibility (e.g., AIM, FIM) will provide valuable data to understand educators' perceptions of the intervention. First, we will examine descriptive of the AIM and FIM measure to determine if the means reached our threshold for defining success (4 or higher out of 5). Next, we will compare item summary scores across participant characteristics to examine for potential patterns. We will focus on the following: (a) investigating variance in outcomes, (b) examining confidence intervals, and (c) assessing for the presence of practically-relevant effects [96-98]. Our targeted sample size will be adequate to provide useful information toward these objectives, pragmatic for recruitment, and consistent with sample sizes of other feasibility studies conducted in early care and education settings [97-101]. We will also use combined data from this study and the ongoing trial (i.e., Table Talk, AFC, BMI, RRS) to compare the 3 conditions ([1] basic WISE, [2] enhanced WISE, and [3] enhanced WISE with deimplementation support) on the targeted outcomes. We will compare confidence intervals, effect sizes, and variance in outcomes across the 3 conditions.

Comparison of data across the three conditions will inform our decision for progression of our deimplementation approach to a full trial. Criteria include (a) similar levels of acceptability and feasibility of the intervention across conditions, (b) qualitative feedback to support moving forward with the de-implementation approach, and (c) a trend toward improved change in feeding practices of the de-implementation condition compared to the implementation only conditions. That is, we would not progress to a full de-implementation trial if educator perceptions of acceptability and feasibility were markedly lower in the de-implementation study compared to the implementation only conditions or if qualitative feedback indicated notable concerns with the de-implementation strategies. Further, we desire to see greater pre to post reductions in use of detrimental feeding practices for the de-implementation condition compared to the implementation conditions to move forward to a full trial.

\section{Discussion}

This study will produce a de-implementation approach that has a basis in the Niven model [56], is reflective of the culture, needs, and desires of ECEs while being consistent with views of administrators. To our knowledge, this will be the first study to apply the EBQI process to de-implementation rather than implementation and may be broadly applicable to the field of IS. Beyond our primary focus on establishing the feasibility and acceptability of our de-implementation approach, the study will provide preliminary data toward 3 predicted outcomes: (1) decreased detrimental feeding practices by ECEs, (2) increased adoption of and fidelity to WISE, and (3) improved child dietary outcomes. These results are expected to contribute to the uptake and sustainability of mealtime interventions to improve the diets of young children. That is, this study will focus on improving the environment in which food is consumed through decreasing detrimental food practices by the ECEs, regardless of what food is available to the children. This focus should facilitate program impact in centers that with a variety of food landscapes, including the two targeted in the current study-one which provides breakfast and lunch for the children and the other which allows for children to either bring their own food or eat in the cafeteria. Results will also apply broadly to the field of IS by informing processes for developing deimplementation approaches in a community, rather than clinical, setting. Thus, this study addresses a significant scientific question with an important public health impact.

Practical considerations for this project include planning for successful stakeholder engagement and addressing the lack of randomization in our study. To improve rapport with ECEs engaged in the EBQI process, we are including mentor panel members from a previous EBQI panel. We expect that including members of similar backgrounds and experiences (including EBQI experience) will increase comfort and trust among new members. These individuals will share their experiences and mentor new panel members. This is a unique innovation for EBQI, and we will share our evaluation of this process with the field. Although the limited number of sites in this study precludes randomization and blinding, our preliminary data (minimum of 2 observations per classroom) suggest that all classrooms have clear room for improvement in detrimental feeding and WISE fidelity. We will have baseline data for each classroom prior to the start of the de-implementation approach, allowing us to examine change from baseline. Finally, participant expectancy could threaten the validity of classroom observations. To counter this, we will use extended assessment periods (i.e., arriving $15 \mathrm{~min}$ early to allow adjustment) as well as multiple observation occasions and multiple methods of assessment (i.e., observed and selfreported feeding).

\section{Conclusions}

The de-implementation approach developed in this study will address detrimental feeding practices and inform de-implementation efforts of other practices in other settings. Specifically, the lessons learned in this 
study will have potential for applicability to future deimplementation efforts of practices that are long-held and culturally ingrained in community settings. Further, the strategy developed in this study may be useful for school-age programs, after school care, and de-implementation aimed at parents.

\section{Acknowledgements}

We would like to acknowledge our community partners who were integral in the development of this protocol and who will be critical to the success of this project.

\section{Authors' contributions}

T.S. led the conception and design of this study in addition to obtaining funding and drafting this manuscript. J.M.R., S.L.J., and G.M.C. contributed to the conception and design of the study and editing of this manuscript. J.P.S. contributed to the design and led the development of the analysis plan. The author(s) read and approved the final manuscript.

\section{Funding}

This project is funded by NIH R03DK117197. Support was also provided in part by the Arkansas Biosciences Institute, the major research component of the Arkansas Tobacco Settlement Proceeds Act as well as the Lincoln Health Foundation. TS also has related salary support from NIH K01 DK110141. The content is solely the responsibility of the authors and does not necessarily represent the official view of the funding agencies.

\section{Availability of data and materials}

The datasets used for this current study will be available from the corresponding author on reasonable request.

\section{Ethics approval and consent to participate}

This protocol was approved by the UAMS Institutional Review Board (IRB 228341). This study was conducted in accordance with all applicable government regulations and University of Arkansas for Medical Sciences research policies and procedures. Waivers of informed consent and Health Insurance Portability and Accountability Act of 1996 (HIPAA) authorization were approved for this project for data collected on adults as this research involves no more than minimal risk to the subjects; waivers will not adversely affect the rights and welfare of the subjects; and the research could not practicably be carried out with the waiver. A passive consent process was approved to allow parents an opportunity for their child to not be included in the de-identified record review (e.g., BMI) and minimally invasive measures (e.g., RRS).

\section{Consent for publication}

Not applicable

\section{Competing interests}

Dr. Taren Swindle and UAMS have a financial interest in the technology (WISE) discussed in this presentation/publication. These financial interests have been reviewed and approved in accordance with the UAMS conflict of interest policies.

\section{Author details}

${ }^{1}$ Department of Family and Preventive Medicine, University of Arkansas for Medical Sciences, 4301 W. Markham St, \#530, Little Rock, AR 72205-7199, USA. ${ }^{2}$ College of Applied and Natural Sciences, Louisiana Tech University, Ruston, USA. ${ }^{3}$ Department of Pediatrics, University of Colorado School of Medicine, Aurora, USA. ${ }^{4}$ College of Public Health, Department of Biostatistics, University of Arkansas for Medical Sciences, Little Rock, USA. ${ }^{5}$ Department of Pharmacy Practice and Psychiatry, University of Arkansas for Medical Sciences, Central Arkansas Veterans Healthcare System, Little Rock, USA.

Received: 21 June 2019 Accepted: 3 November 2020

Published online: 19 November 2020

\section{References}

1. O'Neil A, Quirk SE, Housden S, Brennan SL, Williams L, Pasco JA, et al. Relationship between diet and mental health in children and adolescents: a systematic review. Am J Public Health. 2014;104(10):e31-42. Available from:. https://doi.org/10.2105/AJPH.2014.302110.

2. Marshall S, Burrows T, Collins CE. Systematic review of diet quality indices and their associations with health-related outcomes in children and adolescents. J Hum Nutr Diet. 2014;27(6):577-98. Available from:. https://doi. org/10.1111/jhn.12208.

3. Nyaradi A, Li J, Foster JK, Hickling S, Jacques A, O'Sullivan TA, et al. Goodquality diet in the early years may have a positive effect on academic achievement. Acta Paediatr. 2016;105(5):e209-18. Available from:. https://doi. org/10.1111/apa.13324.

4. Ford CN, Slining MM, Popkin BM. Trends in Dietary Intake among US 2- to 6-year-old children, 1989-2008. J Acad Nutr Diet. 2013;113(1):35-42.e6 Available from: http://www.sciencedirect.com/science/article/pii/S221226 7212014839.

5. Keast D, Fulgoni $V$, Nicklas T, O'Neil C. Food sources of energy and nutrients among children in the United States: national health and nutrition examination survey 2003-2006. Nutrients. 2013;5(1):283-301 Available from: http://www.ncbi.n/m.nih.gov/pubmed/23340318.

6. Aware CC. Child care in America: 2016 state fact cheets. 2016. Available from: http://usa.childcareaware.org/wp-content/uploads/2016/07/2016-FactSheets-Full-Report-02-27-17.pdf.

7. Frisvold D, Lumeng J. Expanding exposure: can increasing the daily duration of head start reduce childhood obesity? J Hum Resour. 2011;46(2):373-402.

8. Matwiejczyk L, Mehta K, Scott J, Tonkin E, Coveney J. Characteristics of effective interventions promoting healthy eating for pre-schoolers in childcare settings: an umbrella review. Nutrients. 2018;10(3):E293 Available from: www.mdpi.com/journal/nutrients.

9. Tysoe J, Wilson C. Influences of the family and childcare food environments on preschoolers' healthy eating. Austrailian J Early Child. 2010;35(3):105-10 Available from: https://search.informit.com.au/documentSummary; $d n=$ 008082004949945;res=IELHSS.

10. Mennella JA, Pepino MY, Reed DR. Genetic and environmental determinants of bitter perception and sweet preferences. Pediatrics. 2005;115(2):e216-22 Available from: http://pediatrics.aappublications.org/content/115/2/e216.abstract.

11. Ventura AK, Worobey J. Early influences on the development of food preferences. Curr Biol. 2013;23(9):R401-8 Available from: http://www. sciencedirect.com/science/article/pii/S096098221300208X.

12. Mennella JA. Ontogeny of taste preferences: basic biology and implications for health. Am J Clin Nutr. 2014;99(3):704S-11S Available from: http://www. ncbi.nlm.nih.gov/pubmed/24452237.

13. Benjamin Neelon SE, Briley ME. Position of the American Dietetic Association: benchmarks for nutrition in child care. J Am Diet Assoc. 2011; 111(4):607-15 Available from: http://www.sciencedirect.com/science/article/ pii/S000282231100191X.

14. Birch LL, McPheee L, Shoba B, Steinberg L, Krehbiel R. "Clean up your plate": effects of child feeding practices on the conditioning of meal size. Learn Motiv. 1987;18(3):301-17 Available from: http://www.sciencedirect.com/ science/article/pii/0023969087900178.

15. Hendy HM, Raudenbush B. Effectiveness of teacher modeling to encourage food acceptance in preschool children. Appetite. 2000;34(1):61-76 Available from: http://www.sciencedirect.com/science/article/pii/S0195666399902866.

16. Orrell-Valente JK, Hill LG, Brechwald WA, Dodge KA, Pettit GS, Bates JE. "Just three more bites": an observational analysis of parents' socialization of children's eating at mealtime. Appetite. 2007;48(1):37-45 Available from: http://www.sciencedirect.com/science/article/pii/S0195666306005137.

17. Hendy HM. Comparison of five teacher actions to encourage children's new food acceptance. Ann Behav Med. 1999;21(1):20-6 Available from: http:// www.ncbi.nlm.nih.gov/pubmed/18425650.

18. Ramsay SA, Branen LJ, Fletcher J, Price E, Johnson SL, Sigman-Grant M. "Are you done?" Child care providers' verbal communication at mealtimes that reinforce or hinder children's internal cues of hunger and satiation. J Nutr Educ Behav. 2010;42(4):265-70 Available from: http://www.sciencedirect. com/science/article/pii/S1499404609002905.

19. Gibson EL, Kreichauf S, Wildgruber A, Vögele C, Summerbell CD, Nixon C, et al. A narrative review of psychological and educational strategies applied to young children's eating behaviours aimed at reducing obesity risk. Obes Rev. 2012;13(Suppl 1):85-95 Available from: http://www.ncbi.nlm.nih.gov/ pubmed/22309067.

20. Fallon M. Exploring self-reported and observed feeding practices of Rhode Island Head Start teachers. Open Access Master's Theses. 2016. Available from: http://digitalcommons.uri.edu/theses/853. 
21. Brown KA, Ogden J, Vögele C, Gibson EL. The role of parental control practices in explaining children's diet and BMI. Appetite. 2008:50(2-3):252-9 Available from: http://www.sciencedirect.com/science/article/pii/S0195666307003315.

22. Dazeley P, Houston-Price C. Exposure to foods' non-taste sensory properties. A nursery intervention to increase children's willingness to try fruit and vegetables. Appetite. 2015;84:1-6 Available from: http://www.sciencedirect. com/science/article/pii/S0195666314004486.

23. Perry LK, Samuelson LK, Burdinie JB. Highchair philosophers: the impact of seating context-dependent exploration on children's naming biases. Dev Sci. 2014;17(5):757-65 Available from: http://www.pubmedcentral.nih.gov/ articlerender.fcgi?artid=4040339\&tool=pmcentrez\&rendertype=abstract.

24. Galloway A, Fiorito L, Francis L, Birch L. "Finish your soup": counterproductive effects of pressuring children to eat on intake and affect. Appetite. 2006;46(3):318-23 Available from: http://www.sciencedirect.com/ science/article/pii/S0195666306000195.

25. Johnson S, Birch L. Parents' and children's adiposity and eating style. Pediatrics. 1994;94(5):653-61 Available from: http://pediatrics. aappublications.org/content/94/5/653.short.

26. Mustonen S, Rantanen R, Tuorila H. Effect of sensory education on school children's food perception: a 2-year follow-up study. Food Qual Prefer. 2009; 20(3):230-40 Available from: http://www.sciencedirect.com/science/article/ pii/S0950329308001353.

27. Reverdy C, Chesnel F, Schlich P, Köster EP, Lange C. Effect of sensory education on willingness to taste novel food in children. Appetite. 2008; 51(1):156-65 Available from: shttp://www.sciencedirect.com/science/article/ pii/S0195666308000718.

28. Batsell WR, Brown AS, Ansfield ME, Paschall GY. "You will eat all of that!": a retrospective analysis of forced consumption episodes. Appetite. 2002;38(3): 211-9 Available from: http://www.sciencedirect.com/science/article/pii/S01 95666301904829.

29. Ward S, Bélanger M, Donovan D, Carrier N. Systematic review of the relationship between childcare educators' practices and preschoolers' physical activity and eating behaviours. Obes Rev. 2015;16(12):1055-70 Available from: http://www.ncbi.nlm.nih.gov/pubmed/26345462.

30. Larson N, Ward D, Neelon S, Story M. What role can child-care settings play in obesity prevention? A review of the evidence and call for research efforts. J Am Diet Assoc. 2011;111(9):1343-62.

31. Birch LL, Zimmerman SI, Hind $H$. The influence of social-affective context on the formation of children's food preferences on JSTOR. Child Dev. 1980; 51(3):856-61 Available from: http://www.jstor.org/stable/1129474?seq= $1 \#$ fndtn-page_scan_tab_contents.

32. Birch L, Marlin D, Rotter J. Eating as the "means" activity in a contingency: effects on young children's food preference. Child Dev. 1984;55(2):431-9 Available from: http://www.jstor.org/stable/1129954.

33. Stark LJ, Collins FL, Osnes PG, Stokes TF. Using reinforcement and cueing to increase healthy snack food choices in preschoolers. J Appl Behav Anal. 1986;19(4):367-79 Available from: http://www.pubmedcentral.nih.gov/ articlerender.fcgi?artid=1308086\&tool=pmcentrez\&rendertype=abstract.

34. Newman J, Taylor A. Effect of a means-end contingency on young children's food preferences. J Exp Child Psychol. 1992;53(2):200-16 Available from: http://www.sciencedirect.com/science/article/pii/002209659290049C.

35. Sanders MR, Patel RK, le Grice B, Shepherd RW. Children with persistent feeding difficulties: an observational analysis of the feeding interactions of problem and non-problem eaters. Health Psychol. 1993;12(1):64-73 Available from: http://europepmc.org/abstract/med/8462502.

36. Wardle J, Herrera M-L, Cooke L, Gibson EL. Modifying children's food preferences: the effects of exposure and reward on acceptance of an unfamiliar vegetable. Eur J Clin Nutr. 2003;57(2):341-8. Available from. https://doi.org/10.1038/sj.ejcn.1601541.

37. Azrin NH, Kellen MJ, Brooks J, Ehle C, Vinas V. Relationship between rate of eating and degree of satiation. Child Fam Behav Ther. 2008;30(4):355-64 Available from: http://www.tandfonline.com/doi/abs/10.1080/073171008024 83223.

38. Gable S, Lutz S. Nutrition socialization experiences of children in the Head Start program. J Am Diet Assoc. 2001;101(5):572-7 Available from: http:// www.sciencedirect.com/science/article/pii/S0002822301001432.

39. Johnson SL. Improving preschoolers' self-regulation of energy intake. Pediatrics. 2000;106(6) Available from: http://pediatrics.aappublications.org/ content/106/6/1429.short.

40. Sigman-Grant M, Christiansen E, Fernandez G, Fletcher J, Johnson S, Branen $L$, et al. Child care provider training and a supportive feeding environment in child care settings in 4 states, 2003. Prev Chronic Dis. 2011;8(5):A113 Available from: http://www.cdc.gov/PCD/issues/2011/sep/10_0224.htm.

41. Kim J, Shim JE, Wiley AR, Kim K, McBride BA. Is there a difference between center and home care providers' training, perceptions, and practices related to obesity prevention? Matern Child Health J. 2012;16(8):1559-66.

42. Sigman-Grant M, Christiansen E, Branen L, Fletcher J, Johnson SL. About feeding children: mealtimes in child-care centers in four western states. J Am Diet Assoc 2008;108(2):340-346. Available from: http://www. sciencedirect.com/science/article/pii/S000282230701797X.

43. Lanigan J. The relationship between practices and child care providers' beliefs related to child feeding and obesity prevention. J Nutr Educ Behav. 2012;44(6):521-9 Available from: http://www.sciencedirect.com/science/ article/pii/S1499404611006063.

44. Freedman M, Alvarez K. Early childhood feeding: assessing knowledge, attitude, and practices of multi-ethnic child-care providers. J Am Diet Assoc. 2010;110(3):447-51 Available from: http://www.sciencedirect.com/science/ article/pii/S000282230901952X.

45. Ward D, Go NAPSACC. Creating an online, interactive tool to improve nutrition environments at child care. In: 9th Annual Conference on the Science of Dissemination and Implmentation in Health. Washington, DC; 2016. Available from: https://academyhealth.confex.com/academyhealth/2 016di/meetingapp.cgi/Paper/13662.

46. Ogata BN, Hayes D. Position of the Academy of Nutrition and Dietetics: nutrition guidance for healthy children ages 2 to 11 years. J Acad Nutri Diet 2014;114(8):1257-76.

47. Prasad V, Ioannidis JP. Evidence-based de-implementation for contradicted, unproven, and aspiring healthcare practices. Implement Sci. 2014;9(1):1 Available from: http://www.ncbi.nlm.nih.gov/pubmed/24398253.

48. Elshaug AG, McWilliams JM, Landon BE. The value of low-value lists. JAMA. 2013;309(8):775 Available from: http://jama.jamanetwork.com/article. aspx?doi=10.1001/jama.2013.828.

49. Morgan D, Wright S. Sanketdhruva. Update on medical overuse. JAMA Intern Med. 2014:E1-5 Available from: https://www.researchgate.net/profile/ Daniel_Morgan8/publication/267753348_Update_on_Medical_Overuse/ links/5640a42208aebaaea1f6885c.pdf.

50. Voorn VMA, Marang-van de Mheen PJ, So-Osman C, Vlieland TPMV, Koopman-van Gemert AWMM, Nelissen RGHH, et al. Designing a strategy to implement cost-effective blood transfusion management in elective hip and knee arthroplasties: a study protocol. Implement Sci. 2012;7(7438):58. Available from: http://www.ncbi.nlm.nih.gov/pubmed/22747693.

51. Gnjidic D, Elshaug AG. De-adoption and its 43 related terms: harmonizing low-value care terminology. BMC Med. 2015;13(1):273 Available from: http:// bmcmedicine.biomedcentral.com/articles/10.1186/s12916-015-0511-4.

52. Murphy MF. The Choosing Wisely campaign to reduce harmful medical overuse: its close association with Patient Blood Management initiatives. Transfus Med. 2015;25(5):287-92 Available from: http://doi.wiley.com/1 $0.1111 /$ tme.12256.

53. Levinson W, Kallewaard M, Bhatia RS, Wolfson D, Shortt S, Kerr EA, et al. 'Choosing Wisely': a growing international campaign. BMJ Qual Saf. 2015 ;24(2): 167-74. Available from: http://www.ncbi.nlm.nih.gov/pubmed/25552584.

54. Elshaug AG, Watt AM, Mundy L, Willis CD. Over 150 potentially low-value health care practices: an Australian study. Med J Aust. 2012;197(10):556-60 Available from: https://www.mja.com.au/journal/2012/197/10/over-150potentially-low-value-health-care-practices-australian-study.

55. Rosenberg A, Agiro A, Gottlieb M, Barron J, Brady P, Liu Y, et al. Early trends among seven recommendations from the choosing wisely campaign. JAMA Intern Med. 2015;175(12):1913 Available from: http://archinte.jamanetwork. com/article.aspx?doi=10.1001/jamainternmed.2015.5441.

56. Niven DJ, Mrklas KJ, Holodinsky JK, Straus SE, Hemmelgarn BR, Jeffs LP, et al. Towards understanding the de-adoption of low-value clinical practices: a scoping review. BMC Med. 2015;13(1):2s55 Available from: http://www. biomedcentral.com/1741-7015/13/255.

57. Swindle T, Phelps J. How does context relate to nutrition promotion and mealtime practice in early care and education settings? A qualitative exploration. J Acad Nutr Diet. 2018;118(11):2081-93.

58. Swindle T, Johnson SL, Davenport K, Whiteside-Mansell L, Thirunavukarasu T, Sadasavin $\mathrm{G}$, et al. A mixed-methods exploration of barriers and facilitators to evidence-based practices for obesity prevention in Head Start. J Nutr Educ Behav. 2019;51(9):1067-79.

59. Swindle T, Johnson SL, Whiteside-Mansell L, Curran GM. A mixed methods protocol for developing and testing implementation strategies for 
evidence-based obesity prevention in childcare: a cluster randomized hybrid type III trial. Implement Sci. 2017;12(1):90.

60. Rubenstein L, Stockdale S, Sapir N, Altman L. A patient-centered primary care practice approach using evidence-based quality improvement: rationale, methods, and early assessment of implementation. J Genet. 2014; Available from: http://link.springer.com/article/10.1007/s11606-013-2703-y.

61. Curran GM, Mukherjee S, Allee E, Owen RR. A process for developing an implementation intervention: QUERI Series. Implement Sci. 2008;3(1):17 Available from: http://implementationscience.biomedcentral.com/articles/1 0.1186/1748-5908-3-17

62. Rubenstein LV, Parker LE, Meredith LS, Altschuler A, dePillis E, Hernandez J, et al. Understanding team-based quality improvement for depression in primary care. Health Serv Res. 2002;37(4):1009-29 Available from: http://doi. wiley.com/10.1034/j.1600-0560.2002.63.x.

63. Brown $\mathrm{CH}$, Curran G, Palinkas LA, Aarons GA, Wells KB, Jones L, et al. An overview of research and evaluation designs for dissemination and implementation. Annu Rev Public Health. 2017;38(1):1-22 Available from: http:// www.annualreviews.org/doi/10.1146/annurev-publhealth-031816-044215.

64. Whiteside-Mansell $L$, Swindle TM. Together we inspire smart eating : a preschool curriculum for obesity prevention in low-income families. J Nutr Educ Behav. 2017; Available from: http://linkinghub.elsevier.com/retrieve/pii/ S1499404617306152.

65. Michie S, Johnston M, Abraham C, Lawton R, Parker D, Walker A, et al. Making psychological theory useful for implementing evidence based practice: a consensus approach. Qual Saf Heal Care. 2005;14(1):26-33 Available from: http://qualitysafety.bmj.com/lookup/doi/10.1136/qshc.2004. 011155.

66. Waltz T, Powell B, Matthieu M, Damschroder L, Chinman M, Smith J, et al. Use of concept mapping to characterize relationships among implementation strategies and assess their feasibility and importance: results from the Expert Recommendations for Implementing Change (ERIC) study. Implement Sci. 2015;10:109 Available from: http://implementationscience.biomedcentral.com/ articles/10.1186/s13012-015-0295-0.

67. Glasgow R. What types of evidence are most needed to advance behavioral medicine? Ann Behav Med. 2008;35:19-25.

68. Gaglio B, Shoup J. The RE-AIM framework: a systematic review of use over time. Am J Dent. 2013; Available from: http://ajph.aphapublications.org/doi/ abs/10.2105/AJPH.2013.301299.

69. Israel BA, Eng E, Schulz AJ, Parker EA. Methods for community based participatory research. Second. San Fransico: Jossey-Bass; 2013.

70. Shea CM, Young TL, Powell BJ, Rohweder C, Enga ZK, Scott JE, et al. Researcher readiness for participating in community-engaged dissemination and implementation research: a conceptual framework of core competencies. Transl Behav Med. 2017;7:1-12 Available from: http://link. springer.com/10.1007/s13142-017-0486-0.

71. Curran GM, Pyne J, Fortney JC, Gifford A, Asch SM, Rimland D, et al. Development and implementation of collaborative care for depression in HIV clinics. AIDS Care. 2011;23(12):1626-36 Available from: https://www. tandfonline.com/doi/full/10.1080/09540121.2011.579943.

72. Fortney J, Enderle M, McDougall S, Clothier J, Otero J, Altman L, et al. Implementation outcomes of evidence-based quality improvement for depression in VA community based outpatient clinics. Implement Sci. 2012; 7(1):30 Available from: http://implementationscience.biomedcentral.com/ articles/10.1186/1748-5908-7-30

73. Smith JL, Williams JW, Owen RR, Rubenstein LV, Chaney E. Developing a national dissemination plan for collaborative care for depression: QUERI Series. Implement Sci. 2008;3(1):59 Available from: http:// implementationscience.biomedcentral.com/articles/10.1186/1748-5908-3-59.

74. Mendel P, Meredith LS, Schoenbaum M, Sherbourne CD, Wells KB. Interventions in organizational and community context: a framework for building evidence on dissemination and implementation in health services research. Adm Policy Ment Health Ment Health Serv Res. 2008;35(1-2):21-37 Available from: http://link.springer.com/10.1007/s10488-007-0144-9.

75. Powell B, Waltz T, Chinman M, Damschroder L, Smith J, Matthieu M, et al. A refined compilation of implementation strategies: results from the Expert Recommendations for Implementing Change (ERIC) project. Implement Sci. 2015;10:21 Available from: https://implementationscience.biomedcentral. com/articles/10.1186/s13012-015-0209-1.

76. Powell BJ, Beidas RS, Lewis CC, Aarons GA, McMillen JC, Proctor EK, et al. Methods to improve the selection and tailoring of implementation strategies. J Behav Health Serv Res. 2017;44(2):177-94.
77. Weiner BJ, Lewis CC, Stanick C, Powell BJ, Dorsey CN, Clary AS, et al. Psychometric assessment of three newly developed implementation outcome measures. Implement Sci. 2017;12(1):108 Available from: http:// implementationscience.biomedcentral.com/articles/10.1186/s13012-0170635-3.

78. Ermakov IV, Gellermann W. Dermal carotenoid measurements via pressure mediated reflection spectroscopy. J Biophotonics. 2012;5(7):559-70. Available from:. https://doi.org/10.1002/jbio.201100122.

79. Scarmo S, Henebery K, Peracchio H, Cartmel B, Lin H, Ermakov I, et al. Skin carotenoid status measured by resonance Raman spectroscopy as a biomarker of fruit and vegetable intake in preschool children. Eur J Clin Nutr. 2012;66(5):555-60. Available from:. https://doi.org/10.1038/ejcn.2012.31.

80. Khoo H, Prasad K, Kong K, Jiang Y, Ismail A. Carotenoids and their isomers: color pigments in fruits and vegetables. Molecules. 2011;16(2):1710-38 Available from: http://www.mdpi.com/1420-3049/16/2/1710.

81. Darvin M, Sterry W, Lademann J, Vergou T. The role of carotenoids in human skin. Molecules. 2011;16(2):1710-38 Available from: http://www. mdpi.com/1420-3049/16/12/10491.

82. Darvin M, Fluhr J, Caspers P, Pool A, Richter H, Patzelt A, et al. In vivo distribution of carotenoids in different anatomical locations of human skin: comparative assessment with two different Raman spectroscopy methods. Exp Dermatol. 2009;18:1060-3 Available from: http://onlinelibrary.wiley.com/ doi/10.1111/j.1600-0625.2009.00946.x/full.

83. Blume-Peytavi U, Rolland A, Darvin ME, Constable A, Pineau I, Voit C, et al. Cutaneous lycopene and $\beta$-carotene levels measured by resonance Raman spectroscopy: high reliability and sensitivity to oral lactolycopene deprivation and supplementation. Eur J Pharm Biopharm. 2009;73(1):187-94 Available from: http://linkinghub.elsevier.com/retrieve/pii/S09396411090014 89.

84. Block $\mathrm{G}$, Subar A. Estimates of nutrient intake from a food frequency questionnaire: the 1987 National Health Interview Survey. J Am Diet Assoc. 1992;92(8):969-77 Available from: http://europepmc.org/abstract/med/164 0041.

85. Freedman LS, Commins JM, Moler JE, Willett W, Tinker LF, Subar AF, et al. Pooled results from 5 validation studies of dietary self-report onstruments using recovery biomarkers for potassium and sodium intake. Am J Epidemiol. 2015;181(7):473-87 Available from: https://academic.oup.com/ aje/article-lookup/doi/10.1093/aje/kwu325.

86. Whiteside-Mansell L, Bradley R, Conners N, Bokony P. The family map: structured family interview to identify risks and strengths in head start families. NHSA Dialog. 2007;10(3-4):189-209 Available from: http://www. tandfonline.com/doi/abs/10.1080/15240750701742239\#.VkYn_XarTmE.

87. Mayne ST, Cartmel B, Scarmo S, Jahns L, Ermakov IV, Gellermann W. Resonance Raman spectroscopic evaluation of skin carotenoids as a biomarker of carotenoid status for human studies. Arch Biochem Biophys. 2013;539(2):163-70. Available from: http://www.sciencedirect.com/science/ article/pii/S000398611300177X

88. Swindle T, Rutledge JM, Dix B, Whiteside-Mansell L. Table Talk: development of an observational tool to assess verbal feeding communications in early care and education settings. Publ Health Nutri. 2017;20(16):2869-77.

89. Fletcher J, Branen L, Price B, Matthews S. Building mealtime environments and relationships. An inventory of mealtime practices for feeding young children in group settings: University of Idaho; 2005. p. 2005.

90. Swindle T, Sigman-Grant M, Branen LJ, Fletcher J, Johnson SL. About feeding children: factor structure and internal reliability of a survey to assess mealtime strategies and beliefs of early childhood education teachers. Int J Behav Nutr Phys Act. 2018;15(1):85 Available from: https://ijbnpa. biomedcentral.com/articles/10.1186/s12966-018-0717-x.

91. Swindle T, Selig J, Rutledge J, Whiteside-Mansell L, Curran G. Fidelity monitoring in complex interventions: a case study of the WISE intervention. Arch Public Heal.

92. Powell BJ, Stanick CF, Halko HM, Dorsey CN, Weiner BJ, Barwick MA, et al. Toward criteria for pragmatic measurement in implementation research and practice: a stakeholder-driven approach using concept mapping. Implement Sci. 2017;12(1):118

93. W3schools. PHP 5 form handling [Internet]. 2017 [cited 2017 Apr 26]. Available from: https://www.w3schools.com/php/php_forms.asp.

94. Hsieh H, Shannon S, et al. Qual Health Res. 2005; [cited 2016 Oct 21]; Available from: http://qhr.sagepub.com/content/15/9/1277.short.

95. Sobo E, Simmes D, Landsverk J, Kurtin P. Rapid assessment with qualitative telephone interviews: lessons from an evaluation of California's Healthy 
Families program \& Medi-Cal for Children. Am J Eval. 2003;24(3):399-408 [cited 2016 Oct 24];: Available from: http://aje.sagepub.com/content/24/3/3 99.short.

96. Dobkin BH. Progressive staging of pilot studies to improve phase III trials for motor interventions. Neurorehabil Neural Repair. 2009;23:197-206.

97. Leon AC, Davis LL, Kraemer HC. The role and interpretation of pilot studies in clinical research. J Psychiatr Res. 2011;45(5):626-9.

98. Thabane L, Ma J, Chu R, Cheng J, Ismaila A, Rios LP, et al. A tutorial on pilot studies: the what, why and how. BMC Med Res Methodol. 2010;10:1.

99. Johnstone A, Hughes AR, Bonnar L, Booth JN, Reilly JJ. An active play intervention to improve physical activity and fundamental movement skills in children of low socio-economic status: feasibility cluster randomised controlled trial. Pilot Feasibility Stud. 2019 5(1):45[cited 2020 Apr 17]. Available from: https://pilotfeasibilitystudies.biomedcentral.com/articles/1 0.1186/s40814-019-0427-4.

100. Rowe A, Titterington J, Taggart L. A classroom-based intervention targeting working memory, attention and language skills in 4-5 year olds (RECALL): study protocol for a cluster randomised feasibility trial. Pilot Feasibility Stud. 2019;5(1):-82 [cited 2020 Apr 17];: Available from: https:// pilotfeasibilitystudies.biomedcentral.com/articles/10.1186/s40814-019-0468-8,

101. Malden S, Reilly JJ, Gibson AM, Bardid F, Summerbell C, De Craemer M, et al. A feasibility cluster randomised controlled trial of a preschool obesity prevention intervention: ToyBox-Scotland. Pilot Feasibility Stud. 2019;5(1):1289 [cited 2020 Apr 17]: Available from: https://pilotfeasibilitystudies. biomedcentral.com/articles/10.1186/s40814-019-0521-7.

\section{Publisher's Note}

Springer Nature remains neutral with regard to jurisdictional claims in published maps and institutional affiliations.

Ready to submit your research? Choose BMC and benefit from:

- fast, convenient online submission

- thorough peer review by experienced researchers in your field

- rapid publication on acceptance

- support for research data, including large and complex data types

- gold Open Access which fosters wider collaboration and increased citations

- maximum visibility for your research: over $100 \mathrm{M}$ website views per year

At $\mathrm{BMC}$, research is always in progress.

Learn more biomedcentral.com/submissions 